Swarthmore College

Works

$5-1-2001$

\title{
Numerical Modeling Of Magnetohydrodynamic Activity In The Swarthmore Spheromak Experiment
}

Vyacheslav S. Lukin , '00

G. Qin

W. H. Matthaeus

Michael R. Brown

Swarthmore College,doc@swarthmore.edu

Follow this and additional works at: https://works.swarthmore.edu/fac-physics

Part of the Physics Commons

Let us know how access to these works benefits you

\section{Recommended Citation}

Vyacheslav S. Lukin , '00; G. Qin; W. H. Matthaeus; and Michael R. Brown. (2001). "Numerical Modeling Of Magnetohydrodynamic Activity In The Swarthmore Spheromak Experiment". Physics Of Plasmas. Volume 8, Issue 5. 1600-1606. DOI: 10.1063/1.1362294

https://works.swarthmore.edu/fac-physics/104

This work is brought to you for free by Swarthmore College Libraries' Works. It has been accepted for inclusion in Physics \& Astronomy Faculty Works by an authorized administrator of Works. For more information, please contact myworks@swarthmore.edu. 


\section{AIP | Physics of \\ Plasmas}

\section{Numerical modeling of magnetohydrodynamic activity in the Swarthmore Spheromak Experiment}

V. S. Lukin, G. Qin, W. H. Matthaeus, and M. R. Brown

Citation: Physics of Plasmas (1994-present) 8, 1600 (2001); doi: 10.1063/1.1362294

View online: http://dx.doi.org/10.1063/1.1362294

View Table of Contents: http://scitation.aip.org/content/aip/journal/pop/8/5?ver=pdfcov

Published by the AIP Publishing

\section{Articles you may be interested in}

Hard magnetohydrodynamic limit in $1 / 3$ sawtooth like activity in LHD

Phys. Plasmas 21, 032501 (2014); 10.1063/1.4867066

Validation of single-fluid and two-fluid magnetohydrodynamic models of the helicity injected torus spheromak experiment with the NIMROD code

Phys. Plasmas 20, 082512 (2013); 10.1063/1.4817951

Three-dimensional magnetohydrodynamics simulations of counter-helicity spheromak merging in the

Swarthmore Spheromak Experiment

Phys. Plasmas 18, 112512 (2011); 10.1063/1.3660533

Magnetohydrodynamic modeling of two-dimensional reconnection in the Magnetic Reconnection Experiment Phys. Plasmas 10, 3131 (2003); 10.1063/1.1591182

Energetic particles and magnetohydrodynamic activity in the Swarthmore Spheromak Experiment

Phys. Plasmas 8, 4816 (2001); 10.1063/1.1405015

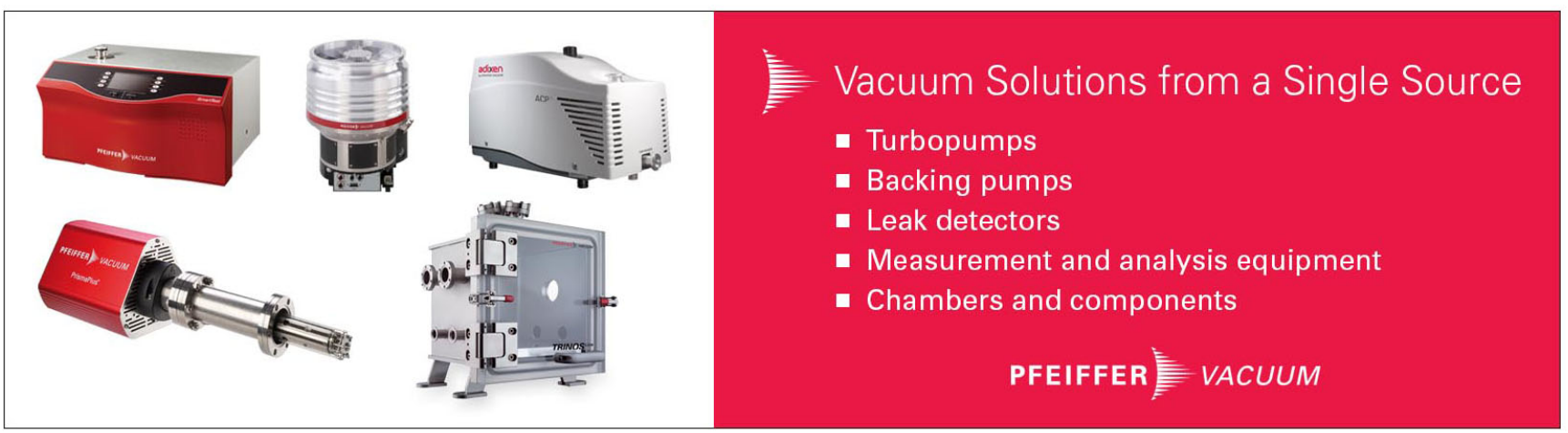




\title{
Numerical modeling of magnetohydrodynamic activity in the Swarthmore Spheromak Experiment
}

\author{
V. S. Lukin ${ }^{\text {a) }}$ \\ Department of Physics and Astronomy, Swarthmore College, Swarthmore, Pennsylvania 19081-1397 \\ G. Qin and W. H. Matthaeus \\ Bartol Research Institute, University of Delaware, Newark, Delaware 19716 \\ M. R. Brown \\ Department of Physics and Astronomy, Swarthmore College, Swarthmore, Pennsylvania 19081-1397
}

(Received 25 September 2000; accepted 13 February 2001)

\begin{abstract}
Results from a three-dimensional axisymmetric resistive magnetohydrodynamic (MHD) simulation are compared to experimental data from the Swarthmore Spheromak Experiment (SSX) [M. R. Brown, Phys. Plasmas 6, 1717 (1999)]. The MHD simulation is run under conditions and with dimensionless parameters similar to the experiment (Lundquist number $S=1000$, plasma beta $\beta$ $=0.1$ ). The simulation is shown to reproduce global equilibrium magnetic field profiles of the spheromaks as well as much of the detailed reconnection dynamics measured when two spheromaks are merged. It is concluded that SSX merger dynamics may be characterized as MHD reconnection, with the likelihood that extensions are needed to account for kinetic effects in the associated current sheet. High spatial and temporal resolution MHD simulation data will be used as input for a particle orbit and energization code. (C) 2001 American Institute of Physics. [DOI: 10.1063/1.1362294]
\end{abstract}

\section{OVERVIEW}

There are several unresolved mysteries related to solar and astrophysical magnetism. First is the origin of solar and astrophysical magnetic fields: the dynamo problem. Second is the role magnetohydrodynamic (MHD) activity plays in plasma heating: the coronal heating problem. Third is the role MHD activity plays in particle acceleration: the cosmic ray problem. There is growing evidence that magnetic reconnection plays a role in all three problems. ${ }^{1}$ The general result of magnetic reconnection is the rapid conversion of magnetic energy to heat and flow energy. Priest and Forbes point out that in the steady state Parker-Sweet model, ${ }^{2,3}$ the conversion is approximately equipartitioned between kinetic flow energy and heat (Priest, p. 126). ${ }^{1}$ Heat can be generated by local twists in the field topology ${ }^{4}$ or at local crossings of converging flux ropes as observed in $\mathrm{x}$-ray bright points on the sun. ${ }^{5}$ Bidirectional Alfvénic outflow jets from a solar flare reconnection site have been observed using a Doppler technique on the Solar Ultraviolet Measurements of Emitted Radiation (SUMER) instrument on the Solar and Heliospheric Observatory (SOHO). ${ }^{6}$ Recently, bidirectional jets were observed in situ in the magnetosphere by a pair of spacecrafts. ${ }^{7}$

The Swarthmore Spheromak Experiment $(\mathrm{SSX})^{8}$ is designed to study magnetic reconnection and particle acceleration due to the controlled, partial merging of two spheromaks (see Fig. 1). SSX is unique in that the spheromaks are generated by plasma guns away from the reconnection zone. ${ }^{9}$ Neutral gas and vacuum magnetic fields are introduced at the guns but only fully ionized plasma and imbedded magnetic

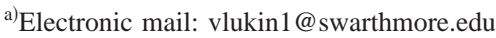

flux convect into the reconnection zone. This affords the possibility in SSX that non-MHD activity is localized near the guns and therefore the subsequent interaction between spheromaks might be described by MHD formalism. In addition, SSX employs a segmented copper boundary at the midplane to allow partial merging as well as allowing each spheromak to relax to its own equilibrium configuration. Early in the discharge we are able to study driven reconnection as the still forming spheromaks merge at the midplane at the Alfvén speed. ${ }^{10}$ Later in the discharge, the two spheromaks separately relax to nearly force-free equilibria ${ }^{9}$ so that we can study spontaneous reconnection. Energetic particles and soft $\mathrm{x}$-rays traverse the field-free, high vacuum gap between flux conservers to various detectors on the midplane.

Other experiments have been designed to study magnetic reconnection and its role in plasma heating. The University of Tokyo experiment TS-3 has focused on " $z-\theta$ " formation for the merging plasma, three-dimensional aspects of reconnection and ion heating. ${ }^{11-14}$ The Princeton Magnetic Reconnection Experiment (MRX) employs a "flux-core" formation scheme and has studied morphology of the reconnection layer, anomalous resistivity and ion heating. ${ }^{15-17} \mathrm{In}$ both cases, vacuum magnetic fields and neutral gas permeate the system and the gas is ionized in situ so that plasma is generated with imbedded magnetic flux (forming the magnetofluid). Typical temporal/spatial scales and plasma parameters in SSX, TS-3, and MRX are all roughly the same: largest scale $=0.5 \mathrm{~m}$, reconnection layer width $\delta \cong 2 \mathrm{~cm}$, $\tau_{\mathrm{Alf}} \cong 3 \mu \mathrm{s}, S \leqslant 1000, n_{e} \cong 10^{14} \mathrm{~cm}^{-3}, T_{e} \cong T_{i}=10-30 \mathrm{eV}$, and $B_{0} \leqslant 1 \mathrm{kG}$.

Initial experiments on SSX mapped out the structure of the MHD equilibrium. ${ }^{9}$ After a turbulent formation phase, we found that the equilibrium was nearly force free, i.e., the 


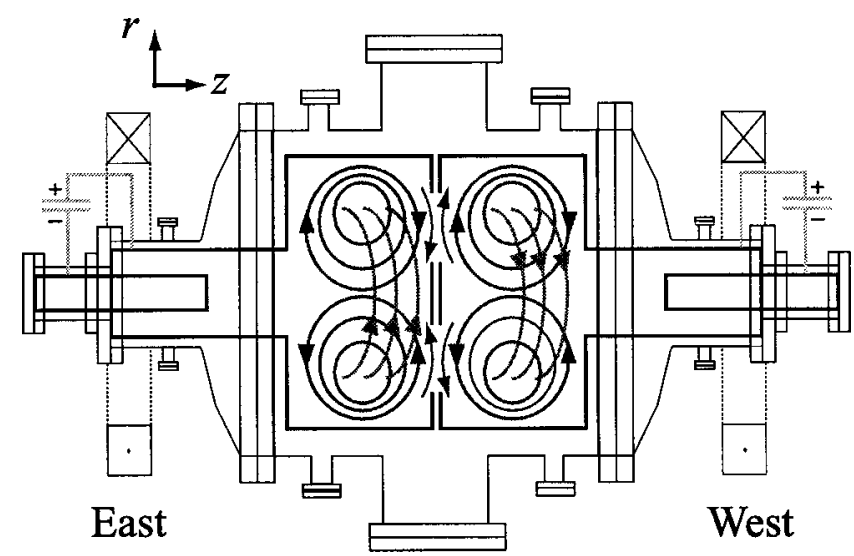

FIG. 1. Swarthmore Spheromak Experiment. Spheromaks are formed by magnetized plasma guns well removed from the reconnection zone. Magnetic fields, energetic particles, and soft x-rays are monitored at the midplane. Magnetic fields, electron density, and temperature as well as impurity emission are monitored in the spheromak equilibria.

magnetic fields obeyed the Taylor eigenvalue equation $\boldsymbol{\nabla} \times \mathbf{B}=\lambda \mathbf{B}$, where $\lambda$ is a constant. Early in the decay phase, $\lambda$ varied by less than $10 \%$ across the machine. Late in the decay, we found departures in the equilibrium from the Taylor state. The toroidal current profile became peaked (due to cooling at the edge) and the magnetic axis shifted outward to larger radius. These departures were well characterized by a $\lambda$ profile quadratic in the poloidal flux $\Phi_{\text {pol }}$.

Reconnection experiments on SSX have shown a number of features. ${ }^{10}$ First, a thin current sheet is formed with a thickness consistent with ion collisionless skin depth $c / \omega_{p i}$ $\cong 2.3 \mathrm{~cm}$, using a typical measured density of $10^{14} \mathrm{~cm}^{-3}$. Second, the magnetic flux in the layer is rapidly annihilated (in about an Alfvén time). Third, this reconnection event is correlated with a burst of plasma flow in the reconnection plane at the Alfvén speed. We have also observed bursts of high energy ions $\left(E \gg M v_{\text {Alf }}^{2} / 2\right)$ and MHD scale fluctuations near the reconnection plane. $8,1,22$

The experimentally observed phenomena in SSX that can be studied using the present approach fall into the broad categories of large scale plasma and magnetic field dynamics, small scale reconnection phenomena and the associated excitation of suprathermal particles. Many MHD simulations have studied these physical phenomena, but the much essential physics can be captured in the very simplified context of periodic two-dimensional MHD. ${ }^{18,21}$ Reconnection simulations of this type can also be employed to study acceleration of test particles under the influence of reconnection electric fields. ${ }^{19-22}$ In these prior numerical studies $\mathbf{B}$ and $\mathbf{v}$ lie in the $x-y$ plane and all field variables are independent of $z$. The electric current density $\mathbf{J}$, magnetic vector potential $\mathbf{A}$, fluid vorticity $\omega$, and electric field $\mathbf{E}$ point in the $\hat{z}$ direction. Typical resolutions were $128 \times 128$ with both magnetic and fluid Reynolds numbers of 1000 . It has been argued $^{21}$ that spectral method simulations with these resolutions and parameter values can accurately portray the onset of turbulent reconnection, in which broad band fluctuations associated with a nonlinear cascade interact strongly with coherent structures that form in the reconnection zone. These turbulent reconnection solutions were employed to compute orbits of batches of test particles, which were observed to reach substantially increased energies. Interpretation of the mechanism of acceleration $^{21}$ noted that a key factor is the interaction of particles with fluctuations near the reconnection zone. This suggests, it may be important to understand details of MHD activity and fluctuations in SSX as well, if we wish to investigate the process of particle energization. In the present work, we will study nonlinear MHD activity using threedimensional (3D) axisymmetric MHD simulations with more realistic boundary conditions and at higher spatial and temporal resolution than in these prior studies. The present simulations are also designed to correspond to the geometry and parameters of the SSX experiment, rather than to study homogeneous MHD activity. Nevertheless, we will see that the basic description of reconnection and MHD activity proceeds in a way that naturally embeds and extends the physics of turbulent reconnection as seen previously. Particle acceleration studies that employ the electromagnetic fields from the MHD simulations are also being employed to study and compare with energization in SSX. These will be described in a subsequent paper.

The paper is organized as follows. In Sec. II, the details of the MHD model are presented as well as the scaling parameters for the TRIM code. In Sec. III, global simulation results of the spheromak equilibrium are compared to early SSX experiments. In Sec. IV, high resolution simulation results in the reconnection zone are compared to recent reconnection experiments.

\section{MHD MODEL AND TRIM}

We employ a 3D cylindrically symmetric nonideal single fluid MHD code-TRIangular Magnetohydrodynamics (TRIM), by Schnack, ${ }^{23}$ to simulate the time-evolution of electromagnetic fields, momentum density, and plasma density in SSX. In doing so, we solve the following set of resistive MHD equations in cylindrical geometry with no slip perfectly conducting boundaries:

$$
\begin{aligned}
& \frac{\partial \rho}{\partial t}+\nabla \cdot(\rho \mathbf{v})=0, \\
& \frac{\partial \mathbf{B}}{\partial t}=\nabla \times(\mathbf{v} \times \mathbf{B})+\frac{\eta}{\mu_{0}} \nabla^{2} \mathbf{B}, \\
& \rho\left[\frac{\partial \mathbf{v}}{\partial t}+(\mathbf{v} \cdot \nabla) \mathbf{v}\right]=\mathbf{J} \times \mathbf{B}-\nabla p+\rho \nu \nabla^{2} \mathbf{v} .
\end{aligned}
$$

In the TRIM implementation, the three components of the vector potential $\mathbf{A}$, the three components of the momentum density $\rho \mathbf{v}$, and the density $\rho$ are propagated in time on an unstructured adaptive grid of triangles in the $\hat{\mathbf{r}} \times \hat{\mathbf{z}}$ plane. Cylindrically symmetric boundary conditions and initial configuration of electromagnetic fields, plasma density, and momentum density, together with several constant parameters, such as the Lundquist number $S=L v_{A} \mu_{0} / \eta$ determine the time evolution of the calculated parameters. ${ }^{23}$ TRIM can potentially be used in a full 3D mode [using a pseudospectral algorithm for the periodic toroidal $(\hat{\boldsymbol{\phi}})$ direction], with or 


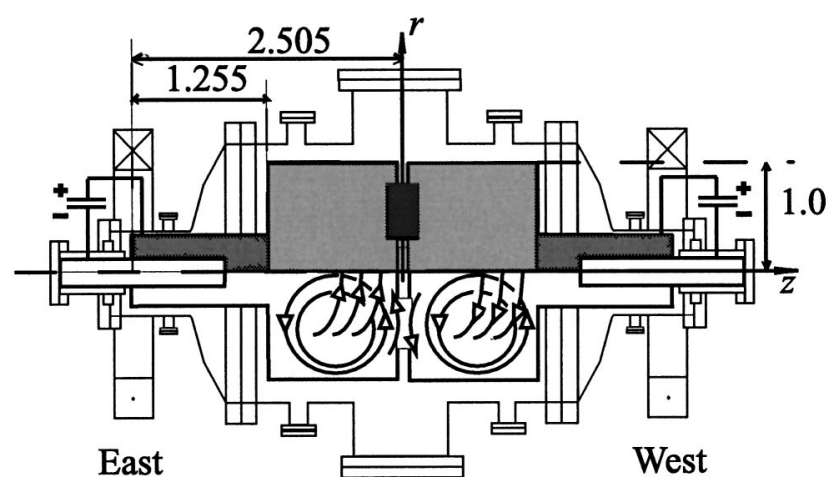

FIG. 2. Computational domain for the TRIM simulation. Axisymmetry is assumed. Computational cell is triagonal. Dimensions are normalized to the flux conserver radius. Reconnection zone has highest resolution; formation guns have intermediate resolution; flux conservers have lowest resolution. Each region is shaded accordingly.

without adaptive mesh, and in several different thermodynamic modes. It also has multiple options for calculating resistivity and viscosity. However, for simplicity we have chosen to run TRIM with the most basic settings: full axisymmetry (cylindrical symmetry), isothermal equation of state, constant uniform dissipation coefficients, and with a fixed mesh (see Fig. 2).

TRIM is written in such a way that in the isothermal mode, pressure $p$ is set to be identically equal to plasma density $\rho$. As a result, the plasma velocity is implicitly calculated in the units of thermal velocity. We choose the magnetic field scale for the simulation $B_{0}=\langle B\rangle=0.05 \mathrm{~T}$ to be the average magnetic field experimentally measured in the SSX flux conserver; the length scale $L_{0}=0.25 \mathrm{~m}$ to be the major radius of the flux conserver and calculate the experimental value of Alfvén velocity to be $v_{\text {Alf }}=\langle B\rangle /\left(\mu_{0} m_{p}\langle n\rangle\right)^{1 / 2}$ $=7.71 \times 10^{4} \mathrm{~m} / \mathrm{s}$, where $\langle n\rangle=2 \times 10^{20} \mathrm{~m}^{-3}$ is the average number density experimentally measured in SSX. Normalizations of all other relevant variables follow from these choices and the specific value assumed for electron temperature $T_{e}$. In particular, the normalizations of plasma density, time, and Lundquist number turn out to depend on $T_{e}$. The Alfvén time $\tau_{\mathrm{Alf}}=L_{0} / v_{\text {Alf }}$ is $3.24 \mu \mathrm{s}$.

We produce a high density mesh on the order of $10^{4}$ points per $L_{0}^{2}$ in the reconnection zone in order to be able to resolve small scale structures in that highly dynamic region (see Fig. 2). Relatively low mesh density (about $5 \times 10^{2}$ points per $L_{0}^{2}$ ) is sufficient in the flux conservers, while in the plasma guns the mesh density of at least $5 \times 10^{3}$ points per $L_{0}^{2}$ is chosen. We have determined that either too fine or too coarse computational grids in the guns give rise to instabilities during the formation stage of the spheromaks. All the physical edges of the layout are assumed to be perfect conductors. Boundary conditions are implemented at the coordinate singularity $r=0$ to ensure a physical solution.

We are unable to introduce external electric fields into the TRIM code and model the fundamentally non-MHD nature of the SSX plasma guns. ${ }^{9}$ However, we can model the spheromak formation process by using MHD processes in the simulation. The configuration of fields used to initialize TRIM is composed of two separate processes. In the experi- ment, we create the poloidal stuffing $B$-field with an external magnetic coil and a highly permeable core inside the inner electrode. In the simulation, we assume existence of a solenoid in the inner electrode of the plasma gun. The magnetic fields produced by such a solenoid can successfully model the corresponding magnetic fields in the experiment. In the experiment, we apply an external voltage between the inner and outer electrodes in order to drive radial current. In the simulation, a gradient in the $z$ direction of the initial toroidal magnetic field $B_{\phi}$ is introduced so that by Ampere's law:

$$
J_{r}=-\frac{\partial B_{\phi}}{\partial z} .
$$

Radial currents are, therefore, induced in the gun and then the $\mathbf{J} \times \mathbf{B}$ forces push the magnetofluid out of the plasma gun, as in the experimental setup. It should be noted that the timing of the experimental and computational spheromak formation methods can disagree considerably due to the fundamental physical differences between an introduction of gradient toroidal magnetic fields into existing magnetofluid and an ionization of neutral hydrogen gas with a high voltage discharge.

One of the greatest challenges we faced in the attempts to reproduce the experimental process of the spheromak formation under the restrictions of resistive MHD was to prevent the code from generating small scale instabilities in the early formation stage in the regions of high poloidal magnetic field right outside of the plasma guns. These problems were overcome by introducing low levels of "background density" in the flux conserver (about 1\%-5\% of the volume averaged plasma density), while the rest of the mass density was placed in the plasma guns in such a way that the density level gradually decays from its peak value in the middle of the gun to the background density level at the gun opening. With these techniques we are able to emulate the startup phase of the spheromak formation with the MHD code.

The simulation results presented below were produced with the following plasma parameters: Lundquist number $S$ $=950$, plasma beta $\beta \approx 0.1$, electron temperature $T_{e}=20 \mathrm{eV}$, and mean density $\langle n\rangle=2.2 \times 10^{20} \mathrm{~m}^{-3}$.

\section{EQUILIBRIUM RESULTS}

Numerical experiments are run from gun formation through spheromak merging, relaxation to equilibrium and finally decay, so we are able to compare numerical and experimental results during all phases of the discharge. The panels of Fig. 3 depict each of these important phases. In Fig. 3, we present color maps of the numerically calculated poloidal flux $\left(\Phi_{\mathrm{pol}}=r A_{\phi}\right)$ at several important times. We define $t=0$ as the time of maximum magnetic energy convected into the reconnection zone (see Fig. 5 below). This choice of origin allows us to effectively compare timings during the MHD-like phases of the SSX discharge, and avoids complications due to the vagaries of the way we have treated the plasma gun dynamics in the startup phase of operation. In Fig. 3(a) $(t=-2.73 \mu \mathrm{s})$, flux and plasma have been ejected out of the gun and are convected towards the reconnection zone at about $v_{\text {Alf }}$. We call this the formation 

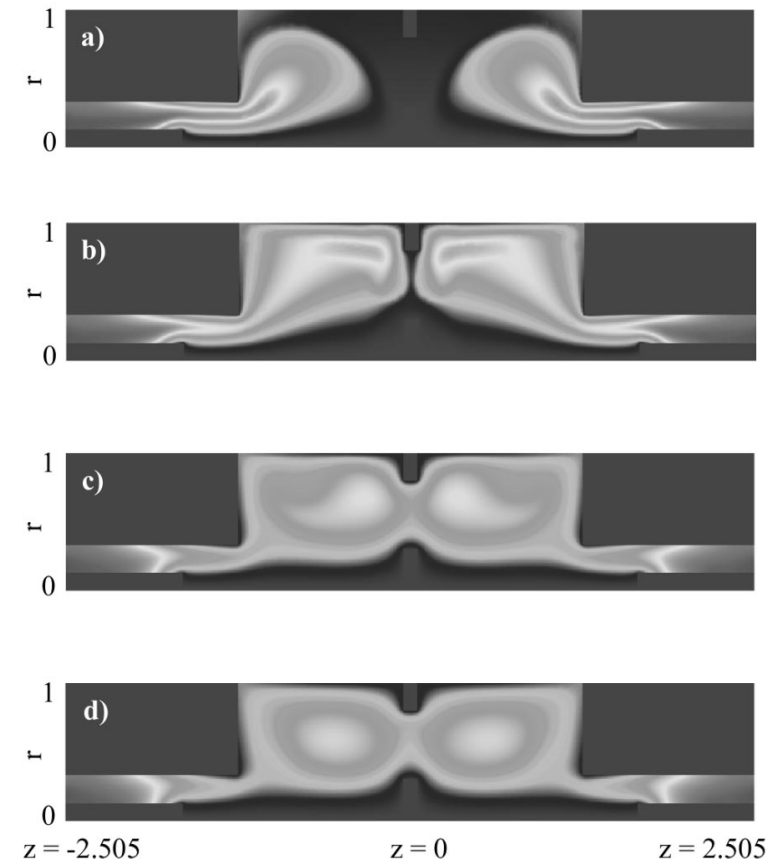

FIG. 3. Evolution of magnetic flux from TRIM simulation. (a) Formation $t=-2.73 \mu \mathrm{s}$, (b) merging $t=0 \mu \mathrm{s}$, (c) relaxation $t=13.5 \mu \mathrm{s}$, (d) equilibrium $t=23.4 \mu$ s. $t=0$ is defined as the time of maximum magnetic energy in the reconnection zone (see Fig. 5). Dimensions are normalized to the radius of the flux conserver.

phase and it corresponds to nearly one Alfvén time before merging. In Fig. 3(b) $(t=0 \mu \mathrm{s})$, oppositely directed poloidal flux has piled up in the reconnection zone and a toroidal current sheet has formed but reconnection has not yet commenced. By $t=13.5 \mu \mathrm{s}$ [Fig. 3(c)], significant driven reconnection has occurred and new flux has convected back into the reconnection zone forming an equilibrium. Most of the interesting reconnection dynamics occur between the initial merging phase $(t=0 \mu \mathrm{s})$ and second pulse of activity around $t=13.5 \mu \mathrm{s}$. By $t=23.4 \mu \mathrm{s}$ the resistive decay phase has begun. This progression of MHD activity will be described further below.

After relaxation and early in the decay phase, the spheromak is well described by a force-free Bessel function equilibrium. ${ }^{9}$ In Fig. 4, we present radial profiles of each component of the magnetic field $\mathbf{B}$ measured at the middle of the flux conserver. Figure 4(a) depicts experimental data from a linear probe array (see Geddes et al., 1998) and Fig. 4(b) depicts numerical data from TRIM, each at about $t$ $=30 \mu \mathrm{s}$. Note that $B_{r}(r)$ is small in both cases and $B_{z}(r)$ (the poloidal field) reverses sign at the magnetic axis $(r$ $\left.=0.6 L_{0}\right) . B_{z}(r)$ and $B_{\phi}(r)$ are well represented by the Bessel functions $J_{0}(r)$ and $J_{1}(r)$, respectively.

We can calculate the correlation between the normalized experimental and simulation data using

$$
\text { Error }=\sqrt{\sum\left(B_{\exp }-B_{\text {sim }}\right)^{2} /\left(\sum B_{\exp }^{2}\right)}
$$

for each component. We find about a $10 \%$ root mean square error for $B_{z}$ and a $25 \%$ root mean square error for $B_{\phi}$. Note that the simulation is not a fit to the data but an $a b$ initio
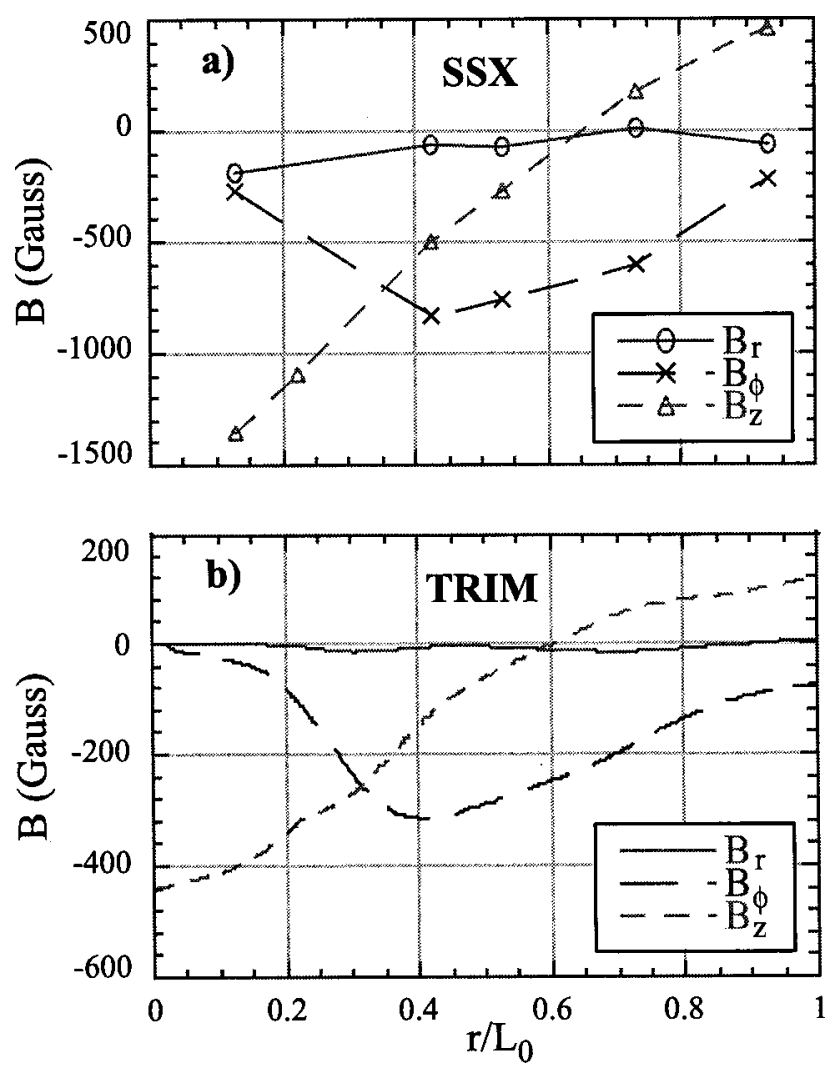

FIG. 4. Radial magnetic field profiles of $B_{r}(r), B_{\phi}(r)$, and $B_{z}(r)$ at the middle of the flux conserver. (a) Experimental data from SSX; (b) simulation data from TRIM. Both profiles were measured during the early decay phase of the nearly force-free equilibria, $t=30 \mu \mathrm{s}$. Dimensions are scaled to the simulation unit length $L_{0}$ (flux conserver radius).

calculation so the correlation is excellent. Corroboration of both temporal and global spatial scales along with relaxation to a force-free equilibrium gives us confidence that TRIM correctly reproduces the global dynamics of the experiment.

\section{RECONNECTION ZONE RESULTS}

The TRIM simulation was performed with highest spatial resolution in the reconnection zone, a $2 \mathrm{D}$ box that straddles the midplane (see Fig. 2). Indeed in the SSX experiment, we focus our diagnostic attention on the reconnection zone since with our geometry, spheromak merging must occur there.

A general picture of the reconnection zone dynamics is given by the time behavior of the magnetic energy. In Fig. 5, we plot the magnetic energy density in the reconnection zone as a function of time $E_{B}(t)$. In Fig. 5(a) we present experimental linear probe data from SSX. ${ }^{10}$ Figure $5(\mathrm{~b})$ shows simulation data from TRIM, in which case $B^{2} / 2 \mu_{0}$ is integrated over a $2 \mathrm{D}$ box defined by $z=+/-3.75 \mathrm{~cm}=+/$ $-0.15 L_{0}$, and $r=13-14.5 \mathrm{~cm}=(0.52-0.58) L_{0}$ in the center of the reconnection zone.

Note, first of all, that magnetic energy is rapidly convected into the reconnection zone and piles up in about one Alfvén time. We call this the merging phase and define $t$ $=0$ as the time of maximum magnetic energy density in the reconnection zone [see Fig. 3(b)]. In roughly another Alfvén 

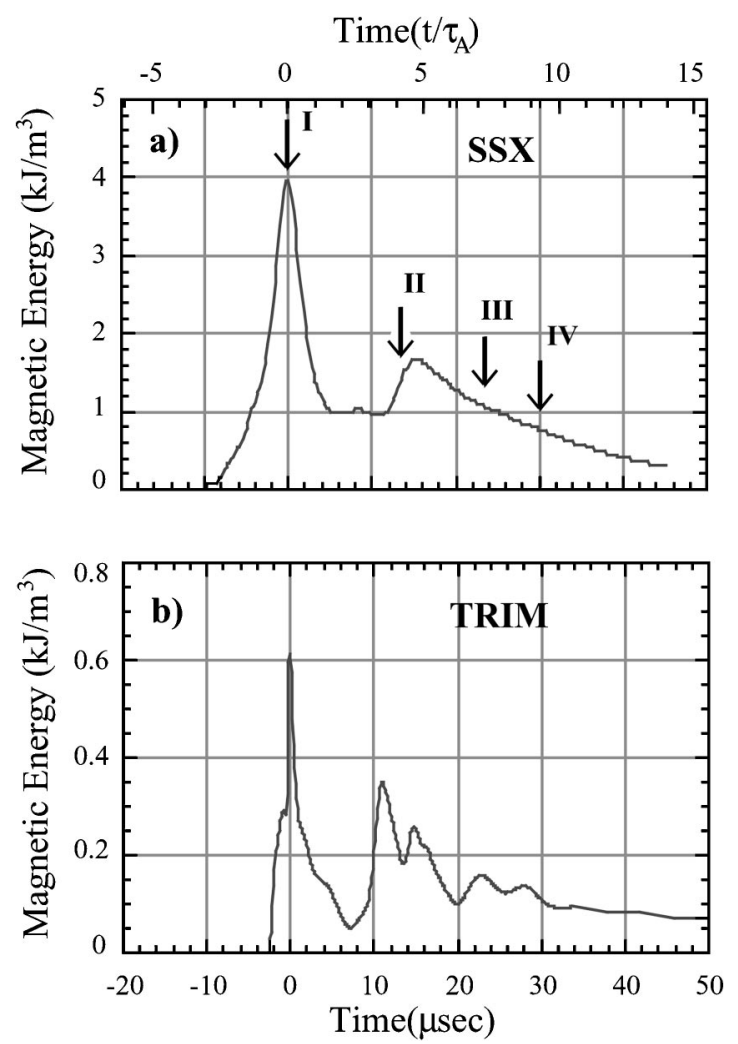

FIG. 5. Evolution of magnetic energy density. Plot of the magnetic energy density $E_{B}(t)$ vs time. (a) Experimental linear probe array data from SSX; (b) simulation data from TRIM, in which case $B^{2} / 2 \mu_{0}$ is integrated over a 2D box defined by $z=+/-3.75 \mathrm{~cm}$ and $r=13-14.5 \mathrm{~cm}$ in the reconnection zone. The four arrows correspond to the last three times of Fig. 3 and the time of Fig. 4. Normalized simulation Alfvén times (top).

time, $3 / 4$ of the magnetic energy is removed from the zone by driven reconnection. There is some indication of a secondary reconnection "event" near $t=13$ to $15 \mu \mathrm{s}$. New flux convects into the layer and the system relaxes to an equilibrium at about $t=20 \mu$ s [corresponding to Figs. 3(c) and 3(d)]. We have indicated the important phases on the figure. Times I, II, and III correspond to initial merging, secondary merging, and relaxation to equilibrium phases [Figs. 3(b), 3(c), 3(d)]. Time IV corresponds to the later time "equilibrium," as seen in Fig. 4 (about $30 \mu \mathrm{s}$ ).

A more detailed picture of the reconnection zone dynamics is given by the time behavior of profiles of $B_{r}(z)$ and $J_{\phi}(z)$. We have made several measurements of these profiles in SSX with 1D probe arrays of various resolutions ${ }^{10}$ as well as $2 \mathrm{D}$ arrays. ${ }^{8}$ In Fig. 6 , we plot $B_{r}(z)$ (the local reconnecting poloidal field) at $t=0 \mu$ s (initial merging) and at $t=23$ $\mu \mathrm{s}$ (equilibrium). In Fig. 7, we plot $J_{\phi}(z)$ [derived from $\left.(\boldsymbol{\nabla} \times \mathbf{B}) / \mu_{0}\right]$ at the same times. (Profiles presented in Fig. 6 and 7 are extracted from the same 2D data. There, we have both the $\hat{\mathbf{r}}$ and $\hat{\mathbf{z}}$ components of $\mathbf{B}$, so the curl can be computed accurately.) Both plots are taken at $r=13.75 \mathrm{~cm}$ $=0.55 L_{0}$ in the middle of the reconnection zone. In Figs. 6(a) and 7(a), we present experimental data from $\mathrm{SSX}^{8}$ and in Figs. 6(b) and 7(b) simulation data from TRIM.

It is clear in the $t=0$ frames of both figures that a reversal of poloidal flux is established at the reconnection layer
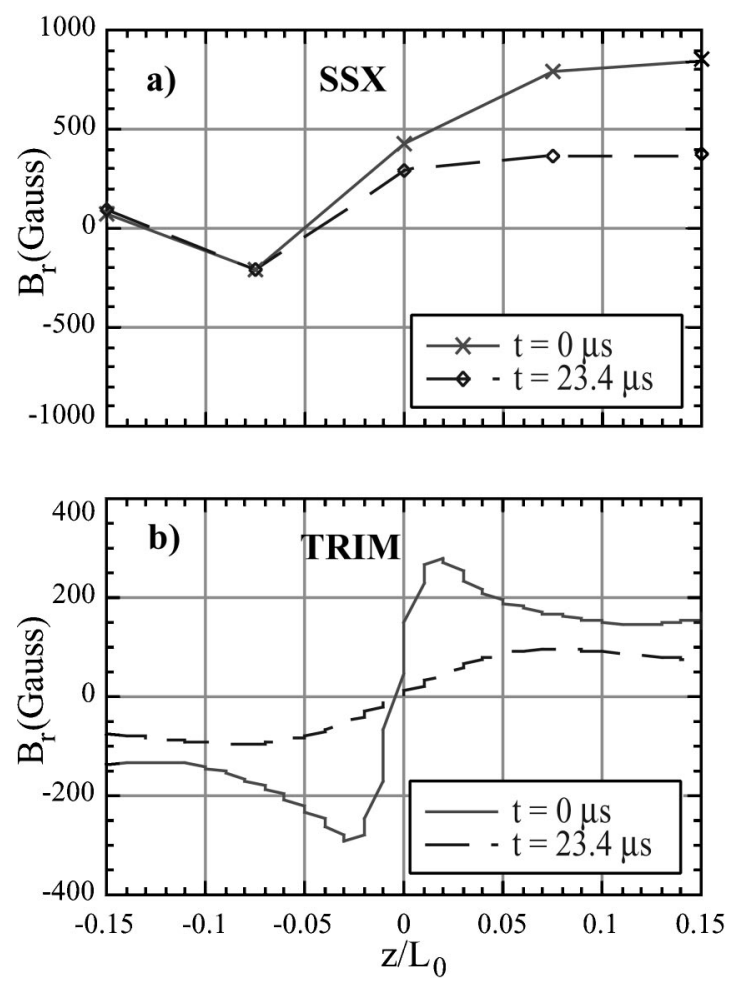

FIG. 6. Reconnection zone magnetic fields. $B_{r}(z)$ through the reconnection zone depicting field reversal at the reconnection layer, (a) experimental data from SSX at $t=0$ (merging) and $t=23.4 \mu$ s (equilibrium); (b) simulation data from TRIM at $t=0$ (merging) and $t=23.4 \mu \mathrm{s}$ (equilibrium). Both cases were measured at $r=13.75 \mathrm{~cm}=0.55 L_{0}$. Dimensions are scaled to the simulation unit length $L_{0}$.

and a current sheet is formed. Later in the decay phase, the gradients have smoothed and the current sheet has broadened. The existence of this dynamical current sheet is direct indication of MHD or MHD-like reconnection activity. It is also interesting to note that in both the SSX and TRIM cases, the current density "overshoots" and reverses sign just beyond the primary interaction region between the regions of oppositely signed magnetic flux. This overshoot is a familiar feature in simulations of highly dynamic reconnection phenomena (see, e.g., Ref. 21). However, notice that even with lower resolution, the experimental data clearly does not exhibit as sharp a gradient in the merging phase as does the simulation data. [Although 2D array data are coarse, finer 1D profiles (see Fig. 4 in Ref. 10) seem to exclude substantial subscale current structure.] All of our experimentally measured reconnection current layers in SSX are a few $\mathrm{cm}$ wide. Note that in the normalized units, $1 \mathrm{~cm}$ corresponds to $1 / 25$ or 0.04 , so our experimentally measured reconnection current layer is about $3.5 \mathrm{~cm}$ (full width at half max) while in the TRIM simulation it is $0.6 \mathrm{~cm}$. We attribute this difference to various kinetic effects, such as the Hall term in Ohm's law and $c / \omega_{p i}$ scaling. ${ }^{10}$ Essentially, plasma ions are redirected by reconnection from axial flow into the layer to radial flow out of the layer. Ion inertia prevents this transition from occurring over distances shorter than $c / \omega_{p i} .{ }^{24} \mathrm{In}$ SSX, $\delta=c / \omega_{p i} \cong 2.3 \mathrm{~cm} \cong 0.1 L_{0}$ for a density of $10^{14} \mathrm{~cm}^{-3}$. Such effects are not included in the MHD simulation. Later in the decay phase, the correspondence between the experi- 

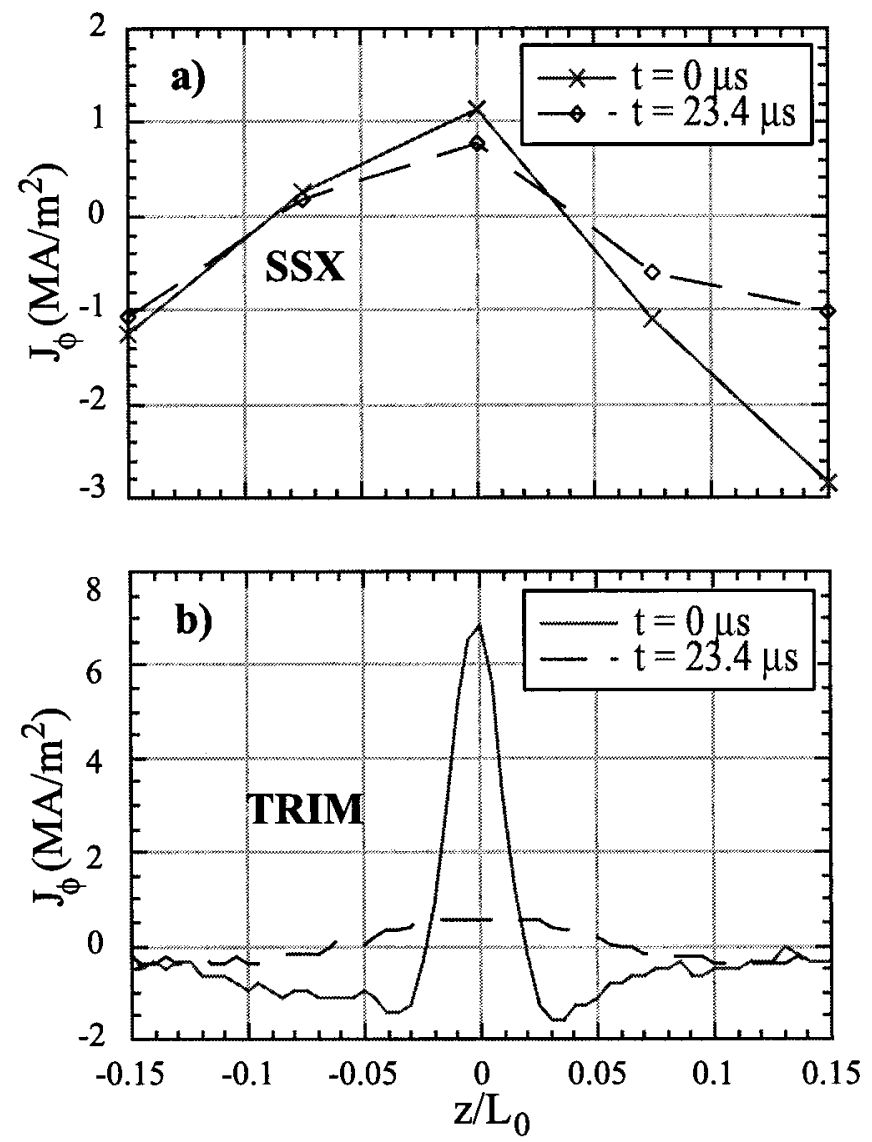

FIG. 7. Reconnection zone current density. $J_{\phi}(z)$ obtained by taking the curl of data corresponding to Fig. 6 (see text). (a) Experimental data from SSX at $t=0$ and $t=23.4 \mu \mathrm{S}$; (b) simulation data from TRIM at $t=0$ and $t$ $=23.4 \mu \mathrm{s}$. Both cases were measured at $r=13.75 \mathrm{~cm}=0.55 L_{0}$. Dimensions are scaled to the simulation unit length $L_{0}$.

ment and the simulation improves significantly $(\delta \cong 2-3 \mathrm{~cm}$ in both cases). This is likely because in both cases the dynamics are controlled by the global equilibria and not local effects.

As a final diagnostic we examine the occurrence of plasma jets generated by reconnection activity and flowing away from the reconnection zone. These are expected to move at approximately the Alfvén speed. ${ }^{1-3}$ We have previously reported Alfvénic jets measured in the SSX experiment. ${ }^{10}$ Gridded energy analyzers are used to measure the ion flux $n_{i} e v$ above some threshold energy escaping the SSX reconnection site. Typically, ion energy analyzers are set to measure all super-thermal ions. In Fig. 8(a), we plot the suprathermal ion flux $n_{i} v_{r}(t)$ for the SSX experiment. ${ }^{10}$ The gridded energy analyzer was located $0.5 \mathrm{~m}$ from the reconnection zone and there was no impediment to the flow. In Fig. 8(b), we plot all the positive radial ion flux $n_{i} v_{r}(t)$ leaving the outer boundary of the reconnection zone of the TRIM simulation. To get a better correspondence to the experiment, we calculate the expected ion flux to a detector 0.5 $\mathrm{m}$ away from the reconnection zone. To more clearly see the correspondence of these measured ion flows to the reconnection activity, the magnetic energy data from Fig. 5 is included in Fig. 8 as well. The peaking of this observed flow just a couple Alfvén times after the onset of intense recon-
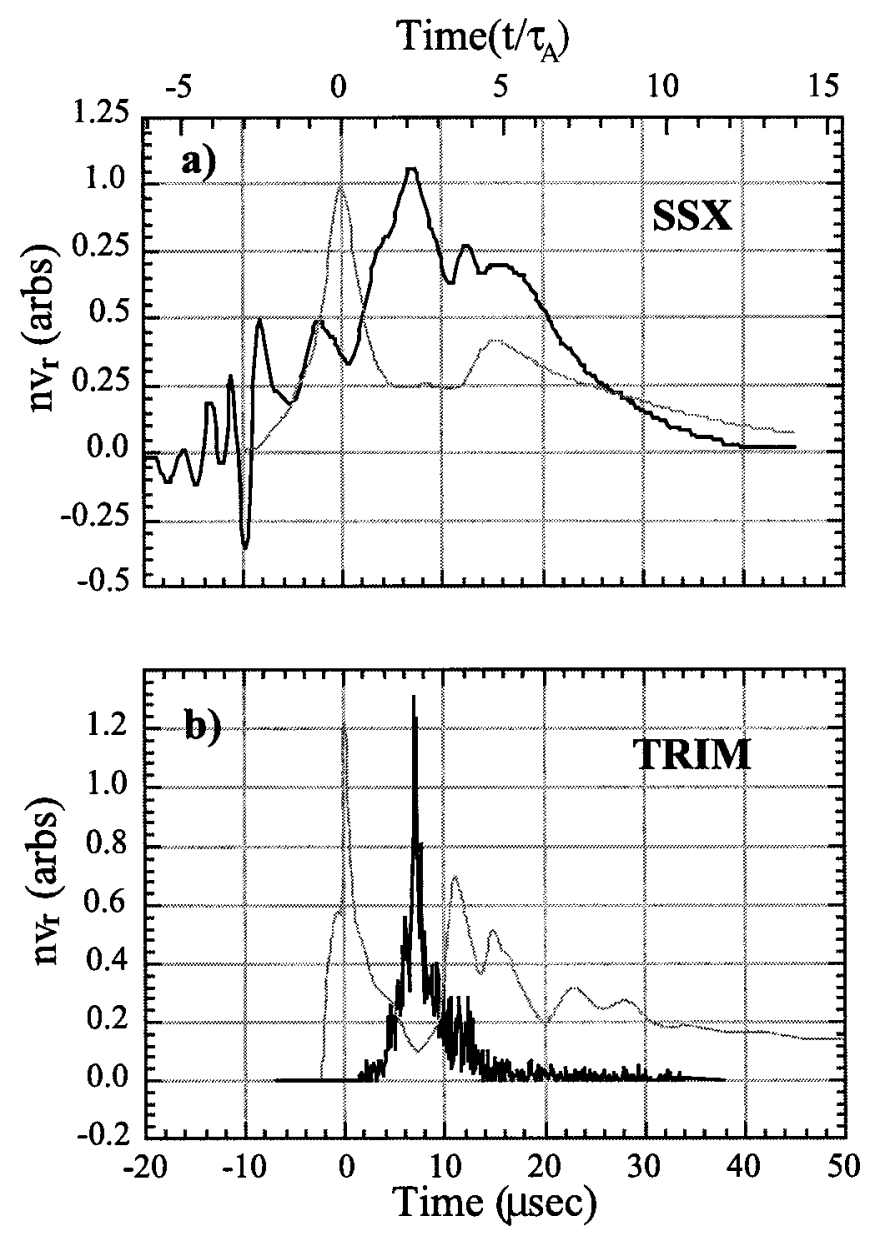

FIG. 8. Alfvénic outflow. Average particle flux $n v_{r}(t)$ flowing out of the reconnection zone. (a) Experimental data from SSX (flux at $r=0.5 \mathrm{~m}$ ); (b) simulation data from TRIM (flux data delayed to $0.5 \mathrm{~m}$ ). The data (bold line) is superimposed over the magnetic energy (shaded line) from Fig. 5. Data are plotted in arbitrary units ("arbs").

nection zone activity is evident in both the experiment and in the simulation. Like the enhancement of magnetic energy, the ion flows decrease in the final phases of the discharge as the equilibrium is approached.

\section{SUMMARY AND CONCLUSIONS}

We examined dynamical activity observed in the SSX experiment and in a numerical simulation using the TRIM code that was designed to represent the experimental situation. The simulation makes use of a simplified one-fluid MHD model, with a simple Ohm's law, a scalar resistivity and viscosity, and an isothermal equation of state. Although a reasonable digital representation of the SSX boundaries were employed in designing the simulation, there are important geometrical differences, and important differences in the startup phase, relative to SSX. The simulation was fully axisymmetric, including the slots in the flux conserver that permit interaction between the spheromaks, while in SSX these slots, cut into the flux limiter, are not fully axisymmetric. In SSX, the spheromak plasma discharges are generated by plasma guns that are decidedly not MHD devices, whereas in the TRIM code we model startup using MHD. To compare 
the simulation and experiment as closely as possible we set the parameters of the TRIM runs to resemble those of SSX. Under these circumstances, perfect correspondence is not envisioned, and the level of similarity between the SSX and TRIM dynamics that we have reported appears to be firm evidence that significant features of SSX dynamics can be reasonably represented by an MHD model.

Our focus has been upon activity associated with the interaction, merger, and relaxation of the two spheromak discharges that are set up within the SSX domain. We have found good correspondence between our 3D axisymmetric MHD simulation and measurements performed on the SSX experiment. Both the global equilibrium and local reconnection effects appear to be closely modeled. This includes, in the merger region, buildup of magnetic energy and electric current density, and the observation of time delayed Alfvénic jets at a distance offset from the region of activity. These diagnostics as well as pictures of magnetic flux activity in TRIM suggest merger and reconnection activity. This leads to relaxation towards an equilibrium state that corresponds qualitatively to a "Bessel function state" that is expected for spheromak relaxation. The one area in which the simulation and experiment appear to differ significantly is with regard to the shape and width of the current channels associated with the reconnection region. This lack of correspondence can be attributed to breakdown of the simple single-fluid MHD model at the shortest scales. In principal, this effect may be at least partially accounted for by including refinements to the Ohm's law, such as by including a Hall term in the induction equation of the present TRIM code. Any further improvements in modeling the reconnection layer in SSX (i.e., $c / \omega_{p i}$ scaling, electron pressure, etc.) would require using two-fluid simulation codes other than TRIM. We intend to pursue these in further studies.

In conclusion, we find evidence that SSX merger dynamics may be characterized as MHD reconnection, with the likelihood that extensions are needed to account for kinetic effects in the current sheet. These findings provide valuable background to our efforts to understand SSX behavior, and more importantly to understand how the dynamical activity seen in SSX may relate to phenomena in solar, space, and astrophysical plasmas. Notably, we now have sufficient confidence in our numerical models to pursue further studies, which will include studies of charged particle dynamics and acceleration in the calculated MHD fields. We plan to com- pare such particle data to improved observation of SSX energetic particles, and to thus further study reconnection as a mechanism for particle acceleration in space and astrophysical contexts.

\section{ACKNOWLEDGMENTS}

M.R.B. is a DOE Junior Faculty Investigator. Special thanks to S. Palmer and D. Radcliff for assistance with the apparatus and D. Schnack for discussions on the TRIM code.

This work was performed under Department of Energy (DOE) Grants Nos. DE-FG02-97ER54422 and ER54490.

${ }^{1}$ E. Priest and T. Forbes, Magnetic Reconnection: MHD Theory and Applications (Cambridge University Press, London, 2000).

${ }^{2}$ E. N. Parker, J. Geophys. Res. 62, 509 (1957).

${ }^{3}$ P. A. Sweet, in Electromagnetic Phenomena in Cosmical Physics, edited by B. Lehnert (Cambridge University Press, London, 1958).

${ }^{4}$ E. N. Parker, Astrophys. J. 174, 499 (1972).

${ }^{5}$ L. Golub, H. Zirin, and H. M. Wang, Sol. Phys. 153, 179 (1994).

${ }^{6}$ D. E. Innes, B. Inhester, W. I. Axford, and K. Wilhelm, Nature (London) 386, 811 (1997).

${ }^{7}$ T. D. Phan, L. M. Kistler, B. Klecker et al., Nature (London) 404, 848 (2000).

${ }^{8}$ M. R. Brown, Phys. Plasmas 6, 1717 (1999).

${ }^{9}$ C. G. R. Geddes, T. W. Kornack, and M. R. Brown, Phys. Plasmas 5, 1027 (1998).

${ }^{10}$ T. W. Kornack, P. K. Sollins, and M. R. Brown, Phys. Rev. E 58, R36 (1998).

${ }^{11}$ M. Yamada, Y. Ono, A. Hayakawa, M. Katsurai, and F. W. Perkins, Phys. Rev. Lett. 655, 721 (1990).

${ }^{12}$ M. Yamada, F. W. Perkins, A. K. MacAulay, Y. Ono, and M. Katsurai, Phys. Fluids B 3, 2379 (1991).

${ }^{13}$ Y. Ono, A. Morita, M. Katsurai, and M. Yamada, Phys. Fluids B 5, 3691 (1993).

${ }^{14}$ Y. Ono, M. Yamada, T. Akao, T. Tajima, and R. Matsumoto, Phys. Rev. Lett. 76, 3328 (1996).

${ }^{15}$ M. Yamada, H. Ji, S. Hsu, T. Carter, R. Kulsrud, Y. Ono, and F. Perkins, Phys. Rev. Lett. 78, 3117 (1997).

${ }^{16}$ H. Ji, M. Yamada, S. Hsu, and R. Kulsrud, Phys. Rev. Lett. 80, 3256 (1998).

${ }^{17}$ S. C. Hsu, G. Fiksel, T. A. Carter, H. Ji, R. M. Kulsrud, and M. Yamada, Phys. Rev. Lett. 80, 3256 (2000).

${ }^{18}$ W. H. Matthaeus and D. Montgomery, J. Plasma Phys. 25, 11 (1981).

${ }^{19}$ W. H. Matthaeus, J. J. Ambrosiano, and M. L. Goldstein, Phys. Rev. Lett. 53, 1449 (1984).

${ }^{20}$ M. L. Goldstein, W. H. Matthaeus, and J. J. Ambrosiano, Geophys. Res. Lett. 13, 205 (1986).

${ }^{21}$ W. H. Matthaeus and S. L. Lamkin, Phys. Fluids 29, 2513 (1986).

${ }^{22}$ J. J. Ambrosiano, W. H. Matthaeus, M. L. Goldstein, and D. Plante, J. Geophys. Res. 93, 14383 (1988).

${ }^{23}$ D. D. Schnack, I. Lottati, Z. Mikic, and P. Satyanarayana, J. Comput. Phys. 140, 71 (1998).

${ }^{24}$ M. A. Shay, J. F. Drake, R. E. Denton, and D. Biskamp, J. Geophys. Res. 103, 9165 (1998). 